\title{
ERRATUM
}

\section{Walking index for spinal cord injury (WISCI II): scale revision}

PL Ditunno and JF Ditunno Jr

Spinal Cord (2009) 47, 349; doi:10.1038/sc.2008.129

Correction to: Spinal Cord (2001) 39, 654-656.

The Author name JF Ditunno Jr was published incorrectly in the above referenced paper.

The correct author list is shown above.

The typesetters apologize for this mistake. 